\title{
Special issue on electromagnetic wave absorbing materials
}

\author{
Hongjing $\mathrm{Wu}^{1, \star}$, Guanglei $\mathrm{Wu}^{2}$, and Biao Zhao ${ }^{3}$ \\ ${ }^{1}$ Northwestern Polytechnical University, Shaanxi, China \\ ${ }^{2}$ Qingdao University, Qingdao, China \\ ${ }^{3}$ Zhengzhou University of Aeronautics, Zhengzhou, China
}

\author{
Published online: \\ 4 September 2021 \\ (C) The Author(s), under \\ exclusive licence to Springer \\ Science+Business Media, LLC, \\ part of Springer Nature 2021
}

With the enormous growth of electromagnetic technology in various fields, the impact of electromagnetic wave radiation on the environment, instruments and human body is increasing. For electronic devices, electromagnetic wave absorbing materials can effectively reduce coupling noise and reduce the interference of electromagnetic wave to internal sensitive components. As an important part of stealth technology, the performance of electromagnetic wave absorbing materials has always been pursued and improved by many countries. The aircraft coated with absorbing material can effectively avoid radar detection. The fairing coated with absorbing material can even reduce the infrared radiation of the engine by nearly $90 \%$. In order to deal with various situations, finding efficient electromagnetic wave absorbing materials has become a major topic in materials science. In recent years, considerable progress has been made in the development of many electromagnetic wave absorbing materials, such as carbon-based materials, magnetic metals/ferrites, ceramics, semiconductors and other absorbing materials.

This special issue publishes a collection of papers that were submitted to the Journal of Materials Science: Materials in Electronics on the theme of electromagnetic wave absorbing materials. Our goal was to continue to provide further knowledge to the readers of this journal and promote the continuous innovation and development of electromagnetic wave absorbing materials.

Publisher's Note Springer Nature remains neutral with regard to jurisdictional claims in published maps and institutional affiliations.

Address correspondence to E-mail: wuhongjing@nwpu.edu.cn 